\title{
RMF+BCS Description of Some Traditional Neutron Magic Isotones
}

\author{
G. Saxena ${ }^{1, a}$, D. Singh ${ }^{2}$, and M. Kaushik ${ }^{2}$ \\ ${ }^{1}$ Department of Physics, Govt. Women Engineering College, Ajmer-305002, India \\ ${ }^{2}$ Department of Physics, University of Rajasthan, Jaipur-302006, India.
}

\begin{abstract}
The traditional neutron magic nuclei with $\mathrm{N}=8,20,28,50,82$ and 126, and those with neutron sub-magic number $\mathrm{N}=40$ are investigated within the relativistic mean-field plus BCS (RMF+BCS) approach. The results indicate appearance of new proton magic numbers as well as the disappearance of conventional magic numbers for nuclei with extreme isospin values. The calculated energies and densities do not indicate any tendency for the proton halo formations in any of the proton rich isotones due to Coulomb interaction and different single particle spectra. However, the potential barrier provided by the Coulomb interaction and that due to the centrifugal force may cause a long delay in the actual decay of proton rich nucleus resulting the extended drip line.
\end{abstract}

\section{Introduction}

Experimental and theoretical studies of exotic nuclei with extreme isospin values are most active areas in the field of nuclear physics in now a days [1]. On the neutron-rich side, exotic phenomena like coupling between bound states and the particle continuum, weak binding of the outermost neutrons, and formation of neutron skins and halos are well known. For proton rich side situation is quite different. Here, nuclei are stabilized by the Coulomb barrier, which tends to localize the proton density in the nuclear interior, thereby preventing the formation of nuclei with large spatial extensions. Recently the relativistic mean field (RMF) theory has been extensively used for the study of unstable nuclei [2-4]. We have applied the RMF model $[5,6]$ for a detailed calculations of the ground state properties of the neutron magic isotones with neutron number $\mathrm{N}=8,20,28,50,82,126$ and the neutron sub-magic isotones with neutron number $\mathrm{N}=40$ using the TMA and the NL-SH force parameterizations.

\section{Theoretical Formulation and Model}

Our RMF calculations have been carried out using the model Lagrangian density with nonlinear terms both for the $\sigma$ and $\omega$ mesons along with the TMA parametrization as described in detail in Refs. [4, 5].

\footnotetext{
ae-mail: gauravphy@gmail.com
} 


$$
\begin{aligned}
\mathcal{L}= & \bar{\psi}\left[l \gamma^{\mu} \partial_{\mu}-M\right] \psi+\frac{1}{2} \partial_{\mu} \sigma \partial^{\mu} \sigma-\frac{1}{2} m_{\sigma}^{2} \sigma^{2}-\frac{1}{3} g_{2} \sigma^{3}-\frac{1}{4} g_{3} \sigma^{4}-g_{\sigma} \bar{\psi} \sigma \psi-\frac{1}{4} H_{\mu \nu} H^{\mu \nu} \\
& +\frac{1}{2} m_{\omega}^{2} \omega_{\mu} \omega^{\mu}+\frac{1}{4} c_{3}\left(\omega_{\mu} \omega^{\mu}\right)^{2}-g_{\omega} \bar{\psi} \gamma^{\mu} \psi \omega_{\mu}-\frac{1}{4} G_{\mu \nu}^{a} G^{a \mu \nu}+\frac{1}{2} m_{\rho}^{2} \rho_{\mu}^{a} \rho^{a \mu}-g_{\rho} \bar{\psi} \gamma_{\mu} \tau^{a} \psi \rho^{\mu a} \\
& -\frac{1}{4} F_{\mu \nu} F^{\mu \nu}-e \bar{\psi} \gamma_{\mu} \frac{\left(1-\tau_{3}\right)}{2} A^{\mu} \psi,
\end{aligned}
$$

where $\mathrm{H}, \mathrm{G}$ and $\mathrm{F}$ are the field tensors for the vector fields and other symbols have their usual meaning. Based on the single-particle spectrum calculated by the RMF described above, we perform a state dependent BCS calculations [7,8]. The continuum is replaced by a set of positive energy states generated by enclosing the nucleus in a spherical box. The well-tested basis expansion method has been employed [9]. For further details of these formulations we refer the readers to ref. $[5,6,9,10]$.

\section{Results and Discussion}

\subsection{Drip-Line Nuclei}

We show in Fig. 1 the variation of proton single particle energy for $\mathrm{N}=40$ isotones as a function of $\mathrm{Z}$. Figure 1 shows that for the proton rich isotones the proton Fermi level (solid circles) lies almost at zero energy. For $Z=40$ the $1 g_{9 / 2}$ state has negative energy and bound. On further addition of two protons $(\mathrm{Z}=42)$, the added proton now occupy $1 \mathrm{~g}_{9 / 2}$ state which now have positive energy and lies at $\epsilon=$ $0.047 \mathrm{MeV}$, whereas proton Fermi energy is seen to be $\epsilon_{f}=-0.732 \mathrm{MeV}$. This state has an appreciable pairing gap akin to bound state and wave function confined in the potential region (not shown here). Therefore this state is considered as a resonant state resulting the bound isotone ${ }_{42}^{82} \mathrm{Mo}_{40}$. The same

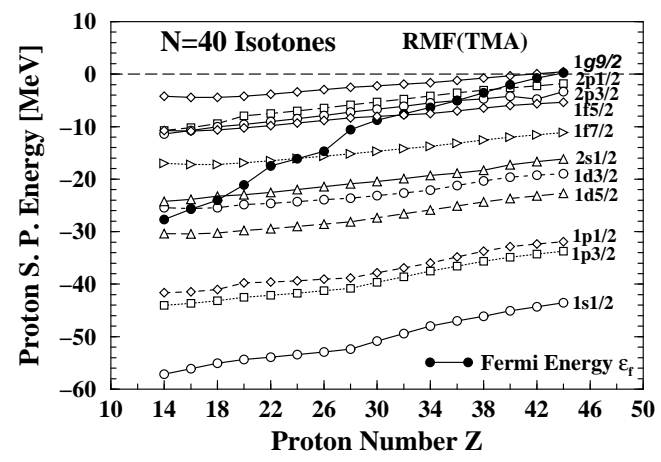

Figure 1. Variation of the ptoton single particle energies for the $N=40$ isotones with increasing proton number $\mathrm{Z}$ for the TMA force.

situation is also found in proton rich $\mathrm{Z}=44$, where $1 \mathrm{~g}_{9 / 2}$ state lies at $\epsilon=0.426 \mathrm{MeV}$, whereas proton Fermi energy is seen to be $\epsilon_{f}=0.237 \mathrm{MeV}$. After $\mathrm{Z}=44$, further addition of two protons now give rise to unbound nuclei, thus one is able to reach up to proton drip line at $\mathrm{Z}=44$ for $\mathrm{N}=40$ isotones $\left({ }_{44}^{84} \mathrm{Ru}_{40}\right)$. This prediction of two proton drip line is in agreement with RHB study by Lalazissis et al. [11]. As a result, we found that near proton drip line contribution of resonant states plays a significant role in deciding the proton drip line for isotonic chains. Moreover, the other isotones with neutron 
INPC 2013

Table 1. Resonant states for drip line isotones

\begin{tabular}{lll}
\hline Isotones & Nuclei & Resonant State \\
\hline $\mathrm{N}=28$ Isotones & ${ }_{30}^{58} \mathrm{Zn}_{28}$ & $1 \mathrm{f}_{5 / 2}$ \\
$\mathrm{~N}=40$ Isotones & ${ }_{42}^{82} \mathrm{Mo}_{40},{ }_{44}^{84} \mathrm{Ru}_{40}$ & $1 \mathrm{~g}_{9 / 2}$ \\
$\mathrm{~N}=$ 50 Isotones & ${ }_{96}^{46} \mathrm{Pd}_{50}$ & $1 g_{7 / 2}$ \\
$\mathrm{~N}=82$ Isotones & ${ }_{72}^{154} \mathrm{Hf}_{82}$ & $1 h_{9 / 2}$ \\
$\mathrm{~N}=126$ Isotones & ${ }_{94}^{220} \mathrm{Pu}_{126}$ & $1 i_{13 / 2}$ \\
\hline
\end{tabular}

number $\mathrm{N}=28,50,82$ and 126 also show the same characteristic and the various resonant states play significant role to give extra stability to the nuclei near or at drip line. In order to save space we show our results in the Table 1 for above mentioned isotones near drip line.

\subsection{Pairing Energy}

Apart from the few nuclei of $\mathrm{N}=40$ isotones, other isotones with $\mathrm{N}=8,20,50,82$ and 126 all are neutron magic and, therefore, for these nuclei the contribution to total pairing energy is mostly from the proton single particle states. For all neutron magic nuclei, the pairing energy vanishes for $\mathrm{Z}$ values corresponding to the proton shell closures. It is seen from the plot for the $\mathrm{N}=8$ isotones in Fig. 2 that

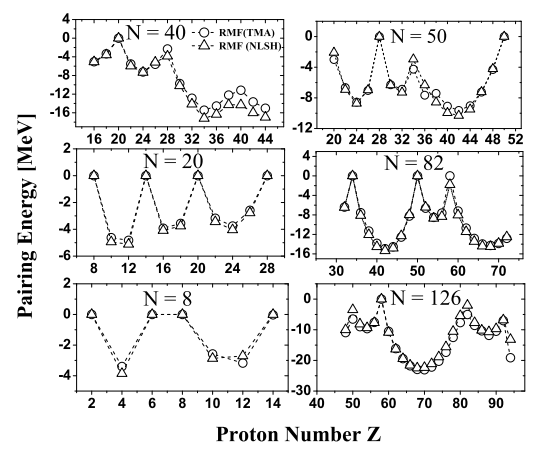

Figure 2. Present RMF results for the pairing energy obtained with the TMA (open circle) are compared with those obtained using the NL-SH (open triangles) force parameters.

the proton shell closure for $\mathrm{N}=8$ isotones occur for $\mathrm{Z}=2,6,8$ and 14 , in which $\mathrm{Z}=6$ and 14 are new shell closure apart from the traditional ones. Similarly, for the $N=20$ isotones proton shell closures observed to occur for $\mathrm{Z}=8,14,20$ and 28 . Whereas in the case of $\mathrm{N}=50,82$ and 126 isotones the value of $\mathrm{Z}$ correspond to the proton shell closures are $\mathrm{Z}=28,50, Z=34,50,58$ and $\mathrm{Z}=58,92$ respectively. However, some neutron rich isotones of $\mathrm{N}=50$ as well as $\mathrm{N}=28$ due to reorganization of single particle states, do not continue to be neutron magic and, hence, there are contributions to the total pairing energy from neutron single particle states as well, as can be seen in the figure where $\mathrm{N}$ $=50$ is not a magic number for $\mathrm{Z}=20$. In addition $\mathrm{N}=126$ does not remain a magic number near proton drip line and give a finite contribution to the total pairing energy. 


\section{EPJ Web of Conferences}

\subsection{Two Proton Separation Energy}

It is seen from the figure of two proton separation energy (Fig. 3), that the calculations using two different force parameters, the TMA and the NL-SH, yield almost identical results for all these isotones and these calculated results for $S_{2 p}$ are in good agreement with the experimental data [12]. An abrupt

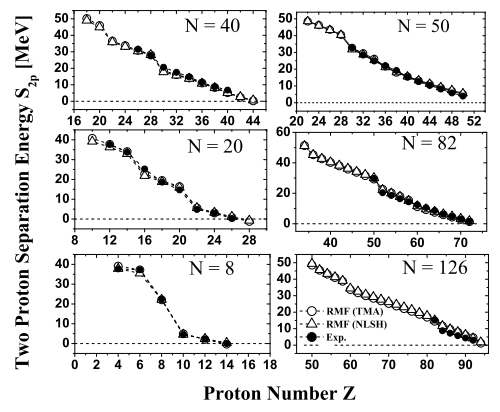

Figure 3. The two proton separation energies calculated with the TMA (open circles) and the NL-SH (open triangle) force parameters are compared the available experimental data[12].

decrease in the $S_{2 p}$ values for the isotones next to magic numbers is clearly seen. The traditional shell closures $Z=8,20,28,50,82$ as well as new shell closures $Z=6,14,34,58$ and 92 can be verified by the Fig. 3. Moreover, for $N=8$ isotones, the two proton drip line is found to occur at $Z=14$ both by the TMA and NL-SH parameters, by experiment and by RHB calculation as well [11]. Similarly, for $\mathrm{N}=20$ isotones drip line is predicted at $\mathrm{Z}=26$ but for $\mathrm{Z}=28$ the two proton separation energy $\mathrm{S}_{2 p}$ becomes negative corresponding to the nucleus ${ }^{48} \mathrm{Ni}$. However, for ${ }^{48} \mathrm{Ni}$ one proton separation energy $\mathrm{S}_{p}$ is found positive. Such nucleus satisfying the condition $\mathrm{S}_{p}>0$ and $\mathrm{S}_{2 p}<0$ may be the possible candidates for simultaneous two-proton emission and are of recent interest [6]. In proton rich nuclei, combined effect of Coulomb barrier and centrifugal barrier prevents the protons from quickly leaving the proton rich nucleus like ${ }^{48} \mathrm{Ni}$ located near or at the two-proton drip-line. The delay associated with the tunneling process allows for the observation of two-proton radioactivity [6].

\section{References}

[1] Rituparna Kanungo, I. Tanihata and A. Ozawa, Phys. Lett. B 512, 261 (2001).

[2] H. Toki, Y Sugahara, D. Hirata, B. V. Carlson, and I. Tanihata, Nucl. Phys. A 524, 633 (1991).

[3] D. Hirata, H. Toki, I. Tanihata, and P. Ring, Phys. Lett. B 314, 168 (1993).

[4] Y Sugahara and H. Toki, Nucl. Phys. A 579, 557 (1994).

[5] H. L. Yadav, S. Sugimoto and H. Toki, Mod. Phys. Lett. A 17, 2523 (2002).

[6] D. Singh and G. Saxena, Int. Jour. Mod. Phys E 21, No.9, 1250076 (2012).

[7] A. M. Lane, Nuclear Theory, Benjamin, (1964).

[8] S. G. Zhou, J. Meng and P. Ring, Phys. Rev. C 68, 034323 (2003).

[9] Y. K. Gambhir, P. Ring and A. Thimet, Ann. Phys. (N.Y.) 198, 132 (1990).

[10] P. Ring, Y. K. Gambhir and G. A. Lalazissis, Comput. Phys. Commun. 105, 77 (1997).

[11] G.A. Lalazissis, D. Vretenar, and P. Ring, Nucl. Phys. A 679, 481 (2001), and references therein.

[12] G. Audi, A. H. Wapstra and C. Thibault, Nucl. Phys. A 729, 337 (2003). 